\title{
Low rectal carcinoma with liver metastasis
}

HCM Hettiarachchi', KB Galketiya ${ }^{2}$, SS Edirimuni ${ }^{3}$

${ }^{1}$ Registrar in Surgery, ${ }^{2}$ Consultant Surgeon, ${ }^{3}$ Senior Registrar in Surgery, Teaching Hospital, Karapitiya, Galle.

\section{Introduction}

$35-45 \%$ of patients with colorectal carcinoma develop hepatic metastases and if left untreated, survival beyond five years is extremely rare. In appropriately selected patients liver resection is associated with a 30-40\% 5-year survival and a $20 \%$ longterm disease free survival.

\section{Case history}

A sixty year-old female, presented with bleeding per rectum and alteration of bowel habits of nine 
month duration. There were no systemic features of malignancy or metastatic disease. She had no family history of colorectal carcinomas.

On abdominal examination there were no masses, hepatosplenomegaly or ascites. Digital rectal examination revealed a circumferential rectal tumour involving anterior half of the rectum $4 \mathrm{~cm}$ from the anal verge.

Rectal biopsy revealed a well differentiated adenocarcinoma. A $3.7 \times 5 \mathrm{~cm}$ size secondary deposit was seen in the right lobe of the liver on ultrasonography. Computerised tomography revealed the lesion to involve segments 5 and 8 of the liver. There was no para-aortic lymphadenopathy.

She was treated with neoadjuvant chemoirradiation. The patient subsequently underwent abdominoperineal resection of rectum and resection of the hepatic metastasis. Right hepatic artery was ligated and divided and right branch of the portal vein was clamped before the procedure. Cholecystectomy and isolated segementectomy of the liver was performed. Ultrasonic dissector and surgical clips were used to achieve haemostasis. Right hepatic vein and IVC were not encircled or clamped during procedure. Patient had an uneventful recovery and was discharged from hospital on the $10^{\text {th }}$ post operative day.

\section{Discussion}

Liver is the commonest site of secondary spread of colorectal cancer. Synchronous hepatic metastases are more aggressive than metachronous lesions. Patients with solitary metastases survive longer than multiple lesions. Patients with unilateral disease live longer than those with bilateral disease.

Diagnosis and staging of the liver metastases is a combination of history, examination and investigations including baseline tests, liver function tests, CEA and imaging techniques including ultrasound scan, CT, and MRI of abdomen. Extrahepatic disease is assessed by chest X-ray and CT thorax.
Liver resection is a safe surgery through understanding of anatomy and physiology combined with modern surgical techniques using ultrasonic dissector and practice of maintaining low venous pressure during the operation.

Hepatic resection is indicated in patients with solitary metastases and in those with metastases confined to localised areas of the liver and if the disease can be macroscopically eradicated with a $5 \mathrm{~mm}$ margin leaving 3 normal segments. Because of the risk of peritoneal seeding, liver biopsy is best avoided in patients with resectable lesions.

If the liver is normal, up to $70 \%$ of the liver can be resected but one must decide on between resecting the entire tumour with an adequate margin, and leaving enough liver for the patient to survive.

The two important prognostic factors following liver resection are; (i) the resection margin (should be at least $5 \mathrm{~mm}$ ) and (ii) the stage of the original primary tumour. Liver transplantation is not recommended for patients with liver metastases, since immunosuppression can cause progression of the disease.

Chemotherapy is recommended for unresectable tumours and radiofrequency ablation improves survival over chemotherapy alone in patients with unresectable hepatic metastases.

\section{References}

1. Charnley RM, Salem T. Colorectal liver metastases: indications for treatment. Surgery International. 2003; 62 : $156-160$

2. Morris PJ Wood WC The colon and rectum In. Alfred M. Cohen. Oxford textbook of surgery. Oxford University press. 2000: 1471-1509.

3. Feliberti EC, Wagman LD. Radiofrequency ablation of liver metastases from colorectal carcinoma. Cancer Control. 2006; 13(1): 48-51. 